\section{A Limbic Encephalitis MR Imaging in a Patient with Behçet Disease and Relapsing Polychondritis}

Behçet disease (BD) is a chronic idiopathic relapsing-remitting multisystem vascular-inflammatory disease characterized by recurrent orogenital ulcerations and uveitis. ${ }^{1}$ Five to ten percent of patients with $\mathrm{BD}$ have clinical evidence of nervous system involvement. The neurologic manifestations of $\mathrm{BD}$ are most commonly due to brain stem or diencephalic involvement. ${ }^{1} \mathrm{MR}$ imaging shows abnormalities in the same areas.

Neurologic involvement has been very rarely reported in relapsing polychondritis (RP). ${ }^{2}$ Mouth and genital ulcers with inflamed cartilage (MAGIC) syndrome includes the clinical manifestations of BD and RP. ${ }^{3}$ Recurrent neurologic disease with MR imaging lesions similar to those seen in $\mathrm{BD}$ has been reported in 1 patient with this syndrome. ${ }^{4}$ We report a patient with BD-RP overlap who had MR imaging findings suggestive of limbic encephalitis. To our knowledge, such an imaging appearance has not been described in $\mathrm{BD}, \mathrm{RP}$, or the MAGIC syndrome.

A 29-year-old man from Somalia was evaluated for 1 week of bilateral eye redness, pain, and markedly decreased vision. His history was remarkable for a 2-year recurrent uveitis. Additional prior symptoms included recurrent oral ulcerations with large-joint arthralgias. He had also noted intermittent swelling, redness, and pain involving the cartilaginous portion of the external ears. His ophthalmologic examination was remarkable for an active panuveitis, markedly decreased visual acuity (left $>$ right), and left-sided cystoid macular edema with papilledema. His neurologic examination was unremarkable except for left-sided hyperreflexia. Brain MR imaging showed increased T2-signal intensity in the mesiotemporal lobes, including the hippocampi (Fig 1A). No abnormal enhancement or restricted diffusion was noted. Also present was an old lacunar infarct in the right ventral pons (Fig 1B). CSF analysis revealed 32 cells $/ \mu \mathrm{L}$, predominantly lymphocytes. CSF protein level was elevated to $60 \mathrm{mg} / \mathrm{dL}$ (normal, $14-45 \mathrm{mg} / \mathrm{dL}$ ), and CSF glucose level was normal. Test results for serum and CSF paraneoplastic antibodies, including neuronal potassium channel antibodies, were negative. CSF microbiologic study findings, including polymerase chain reaction for herpes simplex virus and Tropheryma whipplei, were negative. Human leukocyte antigen (HLA) studies revealed HLA-B51 seropositivity. Because of a suboptimal response to oral and topical steroids, he was started on azathioprine and adalimumab, with marked improvement in his ocular findings.
Our patient had a history of recurrent attacks of oral ulcers, uveitis, arthralgias, and polychondritis. BD is highly prevalent in the eastern Mediterranean region, North Africa, and Eastern Asia. HLA-B51 positivity is strongly associated with the disease in high-prevalence areas. Our patient was from Somalia and had HLA B-51 positivity. Neurologic abnormalities have been well described in $\mathrm{BD}$ and rarely reported in RP. ${ }^{2}$ The type of MR imaging abnormality seen in our patient has not been reported in either. Signal-intensity change in the mesiotemporal lobe suggests a limbic encephalitis, often due to paraneoplastic (including potassium channel-mediated) disease or herpes simplex encephalitis. Other reported causes of mesiotemporal MR imaging lesions include syphilis, Whipple disease, hemorrhagic leukoencephalitis, hypoglycemia, gliomatosis cerebri, lupus erythematosus, primary central nervous system lymphoma, 4-aminopyridine toxicity, and complex partial status epilepticus. ${ }^{5-7}$ BD should also be included in the differential diagnosis of such an MR imaging appearance.

\section{References}

1. Siva A, Saip S. The spectrum of nervous system involvement in Behçet's syndrome and its differential diagnosis. J Neurol 2009;256:513-29 Epub 2009 Apr 27

2. Sundaram MB, Rajput AH. Nervous system complications of relapsing polychondritis. Neurology 1983;33:513-15

3. Firestein GS, Gruber HE, Weisman MH, et al. Mouth and genital ulcers with inflamed cartilage: MAGIC syndrome-five patients with features of relapsing polychondritis and Behçet's disease. Am J Med 1985;79:65-72

4. Gertner E. Severe recurrent neurological disease in the MAGIC syndrome. J Rheumatol 2004;31:1018-19

5. Vieira Santos A, Matias S, Saraiva P, et al. Differential diagnosis of mesiotemporal lesions: case report of neurosyphilis. Neuroradiology 2005;47:664-67

6. Badruddin A, Menon RS, Reder AT. 4-aminopyridine toxicity mimics autoimmune-mediated limbic encephalitis. Neurology 2009;72:1100-1

7. Boeve BF, Bell DG, Noseworthy JH. Bilateral temporal lobe MRI changes in uncomplicated hypoglycemic coma. Can J Neurol Sci 1995;22:56-58

N. Kumar

A.N. Leep Hunderfund Department of Neurology

B.R. Kutzbach

J.S. Pulido

Department of Ophthalmology

G.M. Miller

Department of Radiology

Mayo Clinic

Rochester, Minn

DOI 10.3174/ajnr.A1631

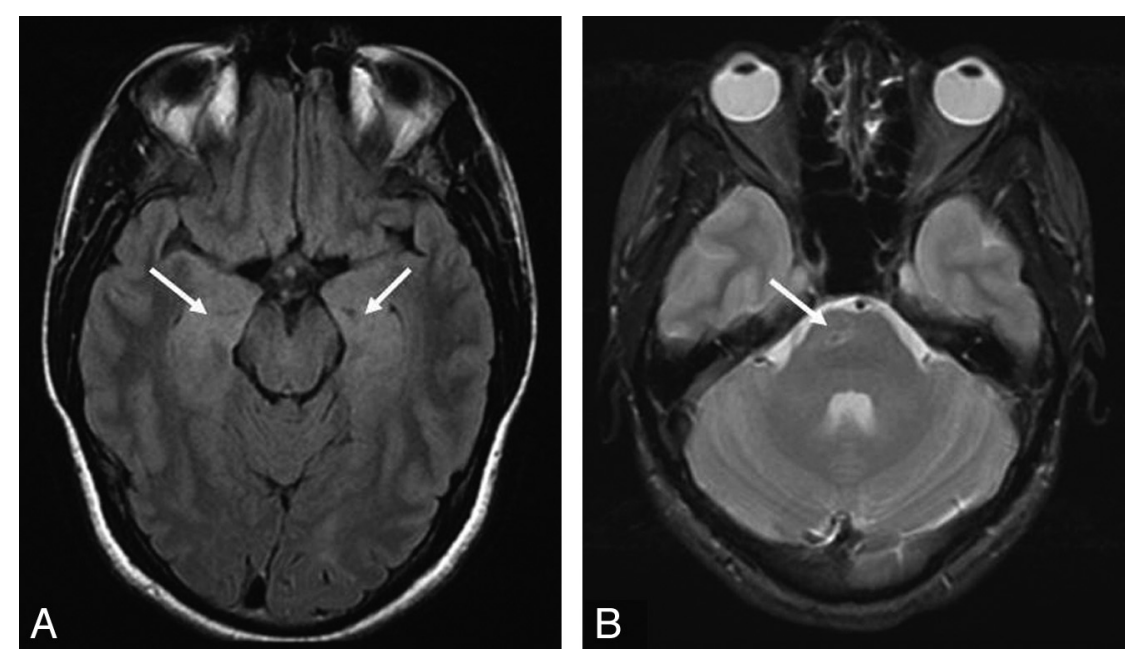

Fig 1. Fluid-attenuated inversion recovery $(A)$ and T2-weighted $(B)$ brain MR images show increased signal intensity in the medial temporal lobe $(A$, arrows $)$ and a right pontine lacune (B, arrow) 